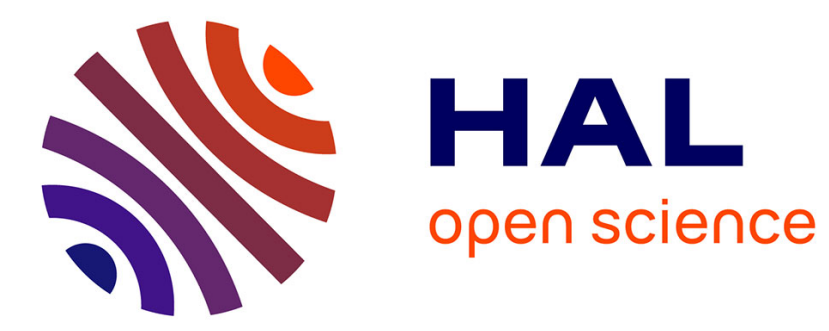

\title{
EEG-fMRI fusion of non-triggered data using Kalman filtering
}

\author{
Thomas Deneux, Olivier Faugeras
}

\section{To cite this version:}

Thomas Deneux, Olivier Faugeras. EEG-fMRI fusion of non-triggered data using Kalman filtering.

[Research Report] RR-5760, INRIA. 2005, pp.11. inria-00070260

\section{HAL Id: inria-00070260 \\ https://hal.inria.fr/inria-00070260}

Submitted on 19 May 2006

HAL is a multi-disciplinary open access archive for the deposit and dissemination of scientific research documents, whether they are published or not. The documents may come from teaching and research institutions in France or abroad, or from public or private research centers.
L'archive ouverte pluridisciplinaire HAL, est destinée au dépôt et à la diffusion de documents scientifiques de niveau recherche, publiés ou non, émanant des établissements d'enseignement et de recherche français ou étrangers, des laboratoires publics ou privés. 
INSTITUT NATIONAL DE RECHERCHE EN INFORMATIQUE ET EN AUTOMATIQUE

\title{
EEG-fMRI fusion of non-triggered data using Kalman filtering
}

\author{
Thomas Deneux — Olivier Faugeras
}

\section{$\mathbf{N}^{\circ} \mathbf{5 7 6 0}$}

Novembre 2005

Thème BIO

\section{apport}

de recherche 



\title{
EEG-fMRI fusion of non-triggered data using Kalman filtering
}

\author{
Thomas Deneux * ${ }^{*}$ Olivier Faugeras ${ }^{\dagger}$ \\ Thème BIO — Systèmes biologiques \\ Projets Odyssée \\ Rapport de recherche $n^{\circ} 5760$ - Novembre 2005 - 11 pages
}

\begin{abstract}
We present a method to combine simultaneous acquisitions of EEG and fMRI measures for estimating ongoing cortical activity. We do not assume that the activity is linked to any repeated stimulation. Rather we solve a very large inverse problem, where EEG and fMRI are noisy measures of the unknown underlying neural activity, and are related to it via realistic physiological models. An extended Kalman filter is used to estimate neural activity from the combined measurements at all instants and all cortical locations. Actually it is already an interesting tool for analyzing EEG or fMRI acquisition in isolation since it is able to handle common difficulties with the temporal aspect of both modalities (temporal smoothness in the EEG inverse problem, and hemodynamic deconvolution in fMRI). Its application to simulated data shows how it takes advantage of EEG high temporal resolution and fMRI spatial precision when reconstructing sources activity.
\end{abstract}

Key-words: fusion, EEG, fMRI, Kalman filter

* thomas.deneux@ens.fr

$\dagger$ olivier.faugeras@sophia.inria.fr 


\section{Fusion de décours temporels EEG et IRMf avec le filtre de Kalman}

Résumé : Nous présentons une méthod pour combiner des acquisitions simultanées en Electroencéphallographie (EEG) et Imagerie par Résonnance Magnétique fonctionnelle (IRMf), en termes d'estimation de l'activité cérébrale sous-jacente. Nous n'estimons pas la réponse à une stimulation particulière répétée au cours d'une expérience, mais le décours temporel de l'activité tout au long de l'acquisition. Cela revient à résoudre un problème inverse de dimensionalité très importante, où l'EEG et l'IRMf sont des mesures bruitées de l'activité neuronale inconnue, à laquelle elles sont reliées par des modèles physiologiques. Nous utilisons un filtre de Kalman étendu pour estimer cette activité en tout instant et en tout point du cortex. Cet outil d'ailleurs se révèle aussi intéressant pour l'analyse de données EEG ou IRMf seules, car il est capable d'intégrer les aspects temporels, en général difficiles à exploiter, des deux modalités (prise en compte d'une continuité temporelle en EEG, et réalisation d'une déconvolution de la réponse hémodynamique en IRMf). Son application sur des données synthétiques montre qu'il tire parti des qualités respective de l'EEG et de l'IRM (bonnes résolutions respectivement temporelle et spaciale).

Mots-clés : fusion, EEG, IRMf, filtre de Kalman 


\section{Introduction}

Recent studies have proved that activation in fMRI can be detected for non-repeated activities, like viewing a movie (1), thus opening the door to trial by trial investigations of cortical activity instead of using evoked potentials or models triggered by a repetitive stimulation. In this context, the possibility to acquire simultaneously EEG and fMRI signals (2) increases the estimation power of cortical activity: the EEG signal is known to bring a much better temporal resolution to fMRI, and it also carries some spatial information if one solves an inverse problem.

Horwitz et al. (3) give an overview of different attempts to combine EEG and fMRI datas. Most existing methods $(4 ; 5)$ do combine the two measures in terms of estimated intensity of the cortical sources, but do not take into account the temporal aspect of acquisition (and thus do not take advantage of the possibility to acquire data simultaneously). Lahaye et al. (6) proposed to use simultaneous EEG-fMRI data to estimate amplitude variations accross trials.

Our approach is to use biophysiological models to relate both electrophysiological and hemodynamic measures to the neural activity $(7 ; 8)$, thereby making possible to estimate the whole sources timecourses from multimodality measures. The mathematical framework is that of Kalman filtering, used by Riera et al. (9) in fMRI, to estimate hidden states timecourses.

\section{Fusion Model}

The main assumption of EEG-fMRI fusion is that both modalities do measure a common cortical activity $u$, that we suppose to be the sum of electrical activities at the surface of the cortex. $u$ is thus a spatiotemporal unknown variable representing the time course of $n_{s}$ sources distributed on the cortex. However, the presence of cerebral activities detected by only one of the two modalities only is permitted by the model, as we will see below.

Let us consider the EEG measure first. We note $y_{\text {eeg }}$ the EEG signal and $n_{e}$ the number of electrode. The EEG model is given by the Maxwell equations, that describe currents propagation from the cortical surface to the scalp (7). Since the propagation times can be neglected, potentials at the electrodes tips at time $t$ are linear functions of the vector of sources activities at the same time, $u(t)$. We add a Gaussian noise that models the measure of activities not detected in fMRI, plus additional measurement and artefact noises:

$$
y_{\mathrm{eeg}}(t)=B u(t)+\eta_{\mathrm{eeg}}(t),
$$

where $B$ is the $n_{e} \times n_{s}$ forward problem matrix, and $\eta_{\text {eeg }}(t) \sim \mathcal{N}\left(0, \Sigma_{\text {eeg }}\right)$.

Let us now note $y_{\mathrm{fmri}}$ the BOLD signal. Its dynamic is described by a biophysiological dynamical system. We use the Buxton Balloon Model (8) as formulated by Friston et al. in (10). This model describes the effects of neural activity on blood flow, venous blood volume, venous deoxyhemoglobin contents and at last BOLD signal. We suppose that the hemodynamic effects related to each cortical source are independent and take place at the

$\mathrm{RR} \mathrm{n}^{\circ} 5760$ 


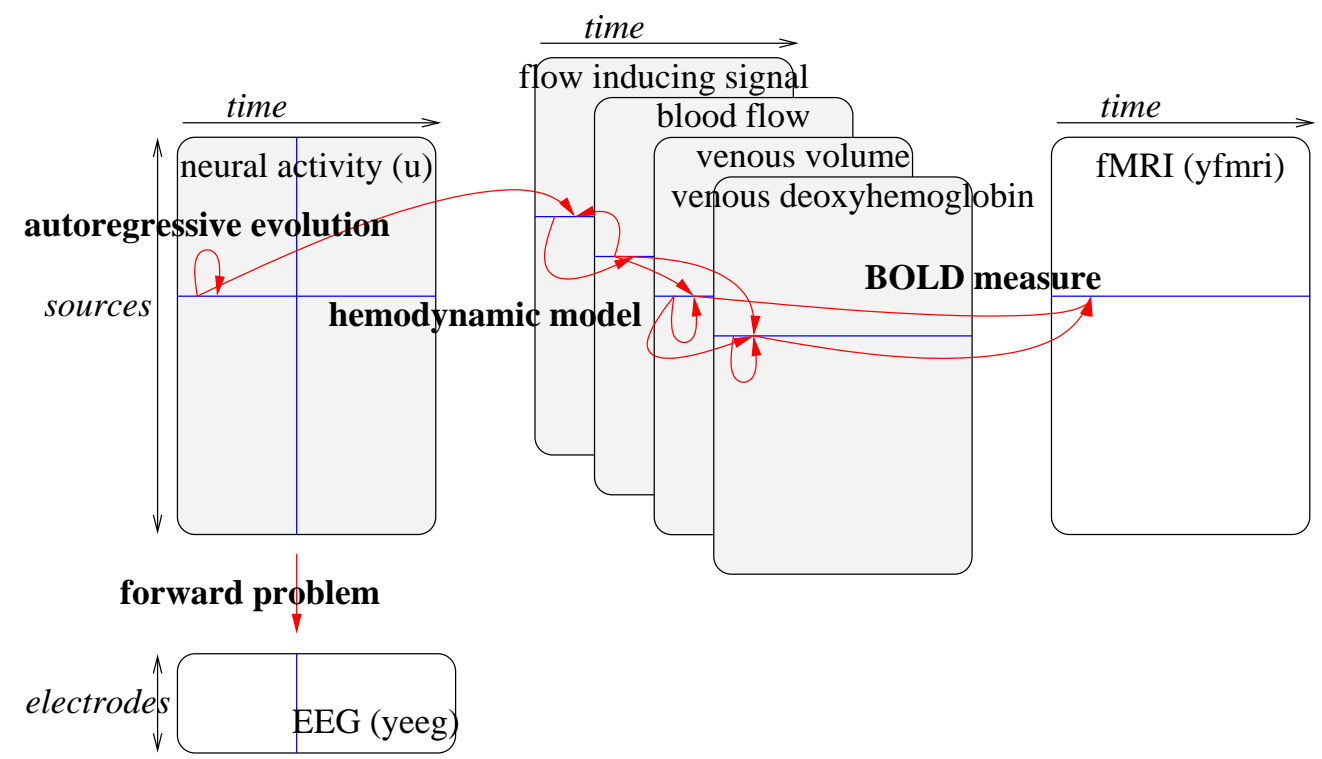

Figure 1: Fusion model. EEG measure is described by the forward problem, while a physiological dynamical system involving neural activity and hidden hemodynamic quantities underlies fMRI measure. Our fusion algorithm estimates the whole spatio-temporal time courses of neural activity, but also of all hemodynamic variables.

same location as the corresponding source. This allows us to describe the fMRI time course at location $s$ independently for each source, as the output of a stochastic dynamical system:

$$
\begin{cases}\dot{x}(s, t) & =f(x(s, t), u(s, t))+\xi_{\text {fmri }}(s, t) \\ y_{\mathrm{fmri}}(s, t) & =g(x(s, t))+\eta_{\mathrm{fmri}}(s, t)\end{cases}
$$

where $x(s, t)$ is the hemodynamic state at location $s$ and time $t$ (its dimension is 4 for the model we use), and $\xi_{\text {fmri }}$ and $\eta_{\mathrm{fmri}}$ are Gaussian evolutive and measurement noises, $\xi_{\text {fmri }}(s, t) \sim \mathcal{N}\left(0, Q_{\text {fmri }}\right)$ and $\eta_{\mathrm{fmri}}(s, t) \sim \mathcal{N}\left(0, \sigma_{\mathrm{fmri}}^{2}\right)$. Evolutive noise $\xi_{\mathrm{fmri}}$ implicitly takes into account cortical activities not detected in EEG.

In order to get a posteriori probabilities on the sources time courses $u$ with the Kalman filter, we need to set a priori probabilities on them. We choose to use the simple autoregressive model:

$$
\dot{u}(t)=u(t)+\xi_{u}(t)
$$

where $\xi_{u}$ is a Gaussian evolutive noise $\xi_{u}(t) \sim \mathcal{N}\left(0, Q_{\mathrm{u}}\right)$. The spatial correlation matrix between sources $Q_{\mathrm{u}}$ is constructed according to geometrical constraints, to ensure spatial smoothness of the cortical activity. Temporal smoothness is ensured by the autoregression, and can be regulated through the diagonal terms in $Q_{\mathrm{u}}$. 


\section{Algorithm}

The whole model described above can be expressed as a single nonlinear dynamical system, which we write in discrete form:

$$
\begin{cases}X_{k+1} & =F\left(X_{k}\right)+\xi_{k} \\ Y_{k} & =G\left(X_{k}\right)+\eta_{k}\end{cases}
$$

$X_{k}$ is the set of hidden state variables (sources activities $u$ and hemodynamics states $x$ ) of all sources at instant $k$, concatenated in a single vector.

$F$ gathers the evolution equations in (2) and (3).

$Y_{k}$ is the set of measurements (EEG signals at all electrodes and/or fMRI signals at all sources locations), concatenated in a single vector. Since there are much less acquisition times in fMRI than in EEG, we interpolate the fMRI time course to all EEG instants using a linear interpolation.

$G$ gathers the measurement equations in (1) and (2) .

At last, $\xi_{k}$ and $\eta_{k}$ are Gaussian evolutive and measurement noises, $\xi_{k} \sim \mathcal{N}(0, Q)$ and $\eta_{k} \sim \mathcal{N}(0, R)$; their variances are calculated from the values of $Q_{\mathrm{u}}, \Sigma_{\text {eeg }}, Q_{\mathrm{fmri}}$ and $\sigma_{\text {fmri }}^{2}$.

Given the measure $Y$, we can estimate the hidden state $X$ (and in particular $u$, the neural activity detected by EEG and fMRI) recursively for increasing times, by using an extended Kalman filter $(11 ; 12)$ : if both evolutive and measure noises are Gaussian, and if at each sampling instant the nonlinear evolution and measure functions are approximated up to first order, then the a posteriori distribution of the hidden states is also Gaussian; the Kalman filter calculates the mean and variance of this distribution.

To be strictly accurate, the estimation of hidden states at instant $k$ given by the Kalman filter does not take into account measures after instant $k$. It calculates the following means and variances:

$$
\begin{aligned}
\hat{X}_{k}^{k} & =E\left(X_{k} \mid Y_{1} \ldots Y_{k}\right) \\
P_{k}^{k} & =E\left(X_{k} X_{k}^{T} \mid Y_{1} \ldots Y_{k}\right) .
\end{aligned}
$$

Then, it is necessary to apply a Kalman smoother (11) to obtain the final a posteriori distribution

$$
\begin{aligned}
\hat{X}_{k}^{T} & =E\left(X_{k} \mid Y_{1} \ldots Y_{T}\right) \\
P_{k}^{T} & =E\left(X_{k} X_{k}^{T} \mid Y_{1} \ldots Y_{T}\right),
\end{aligned}
$$

where $T$ is the number of instants.

Details of the Kalman filter and smoother are given in the appendix.

\section{Artificial data}

We present simulation results, using a realistic head model based on the segmentation of anatomical MRI data. The number of sources on the cortex is currently limited by memory

$\mathrm{RR} \mathrm{n}^{\circ} 5760$ 

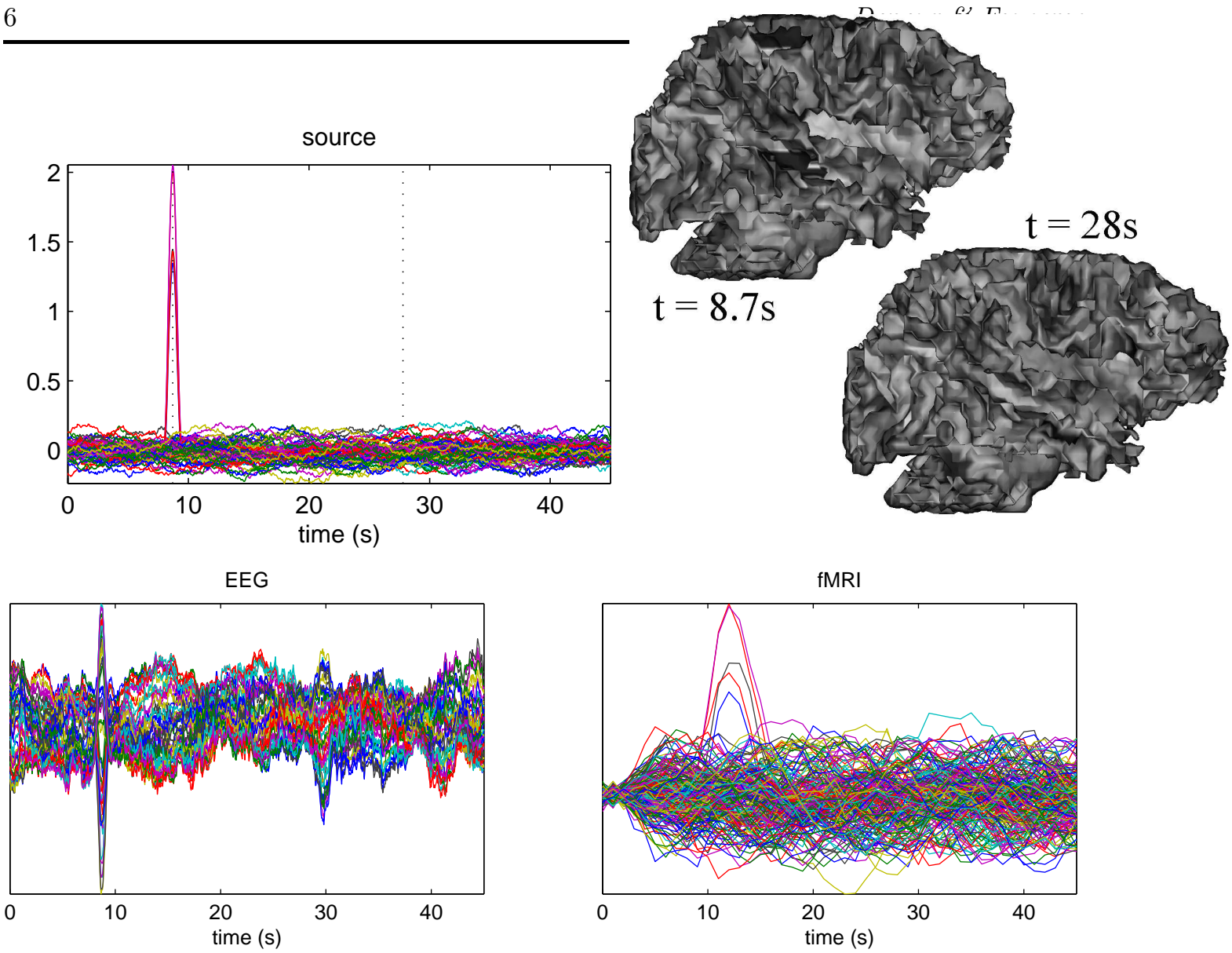

Figure 2: Artificial data. Top: (left) plot of the time courses of all cortical sources; neural activity was generated according to the evolutive model (3) and a rapid burst (1s half width) was added to a few sources; (right) activity mapped on the cortex at instants represented by dotted vertical lines. Bottom: EEG and fMRI measures generated by the forward problem (1) and the Balloon Model (2).

constraints: indeed, the hidden-states dimension in the model (the length of vector $\hat{X}_{k}$ ) is equal to the number of sources $n_{s}$ times the number of variables for one source $(u$ and 4 hemodynamic variables in $x)$, and the algorithm uses square matrices of this size $\left(P_{k}^{k-1}, P_{k}^{k}\right.$, $J$... see appendix). Thus we use a model with 1000 sources on the cortex. Our decimation method allows us to interpolate between these 1000 sources and the 45000 vertices of the cortex full mesh, from which we construct the EEG forward problem matrix $B$ in (1). We used a set of 64 electrode locations on the scalp.

We simulated cortex activity according to the evolutive model (3), and added some wave signals to a few chosen sources, to test the model ability to handle fast and strong variations. 
Then the EEG and fMRI signals were simulated according to the models ((1) and (2)), see figure 2. Physiological parameters were assigned plausible values (10).

\section{Results}

The current version of the algorithm can work whith EEG or fMRI measures alone, or with both ot them simultaneously. To emphasize the improvement brought by the fusion, we first ran the algorithm on each modality separately. Figure 3 shows the results for EEG, fMRI and EEG+fMRI estimations. The respective qualities of EEG and fMRI in term of temporal and spatial resolutions are clearly illustrated. Indeed, in the EEG estimation, the temporal pattern of activation peak around $\mathrm{t}=8.5 \mathrm{~s}$ is recovered exactly, but its localisation is quite diffuse (more sources are activated than in the original data, and the activation power is reduced). On the other hand, in the fMRI estimation, it is the estimated time course that is diffuse, whereas the method found the right activation focus. The fusion algorithm then finds a compromise between the two estimations, and the smoothness of neural sources time courses. It was not able to find the exact amplitude of the activity peak as in figure 2, but this is not surprising since the algorithm used an a priori auto-regressive model for sources activity (equation (3)), that supposes a temporal smoothness.

Figure 4 shows the estimated time courses of a few cortical sources with different characteristics. When it is applied to fMRI only, the Kalman filter performs a deconvolution of the BOLD signal for each source location, that leads to a quite acurate estimation already (center row in figure 3 and cyan time courses in figure 4). Compared to it, the EEG estimation (top row in 3 and green time courses in 4) is much worse, due to the ill-posedness of the inverse problem. However, in the fusion context (bottom row in 3 and red time courses in 4), EEG brings a complementary information in terms of rapid fluctuations, leading to a better estimation of ongoing neural activity.

\section{Conclusion}

Our work illustrates how information coming from simultaneous EEG and fMRI measures can be integrated together to obtain a better estimation of the underlying neural activity. Applied to the EEG alone, the Kalman filter technique solves an inverse problem that constrains the cortical sources to be smooth in time. Applied to fMRI alone, it performs a signal deconvolution. Applied to both simultaneously, it integrates temporal and spatial aspects of each modality, and takes advantage of their respective qualities.

In our simulation data, it was particularily efficient to estimate neural "background activity". It could thus open the door to neural activity estimations without averaging over repetitions of a same stimulation.

We are currently using the method on simultaneous EEG and fMRI acquisition on epileptic subjects, which will allow us also to test and possibly correct the model main hypothesis: the existence of a common neural activity that underspin EEG and fMRI measurements,

$\mathrm{RR} \mathrm{n}^{\circ} 5760$ 

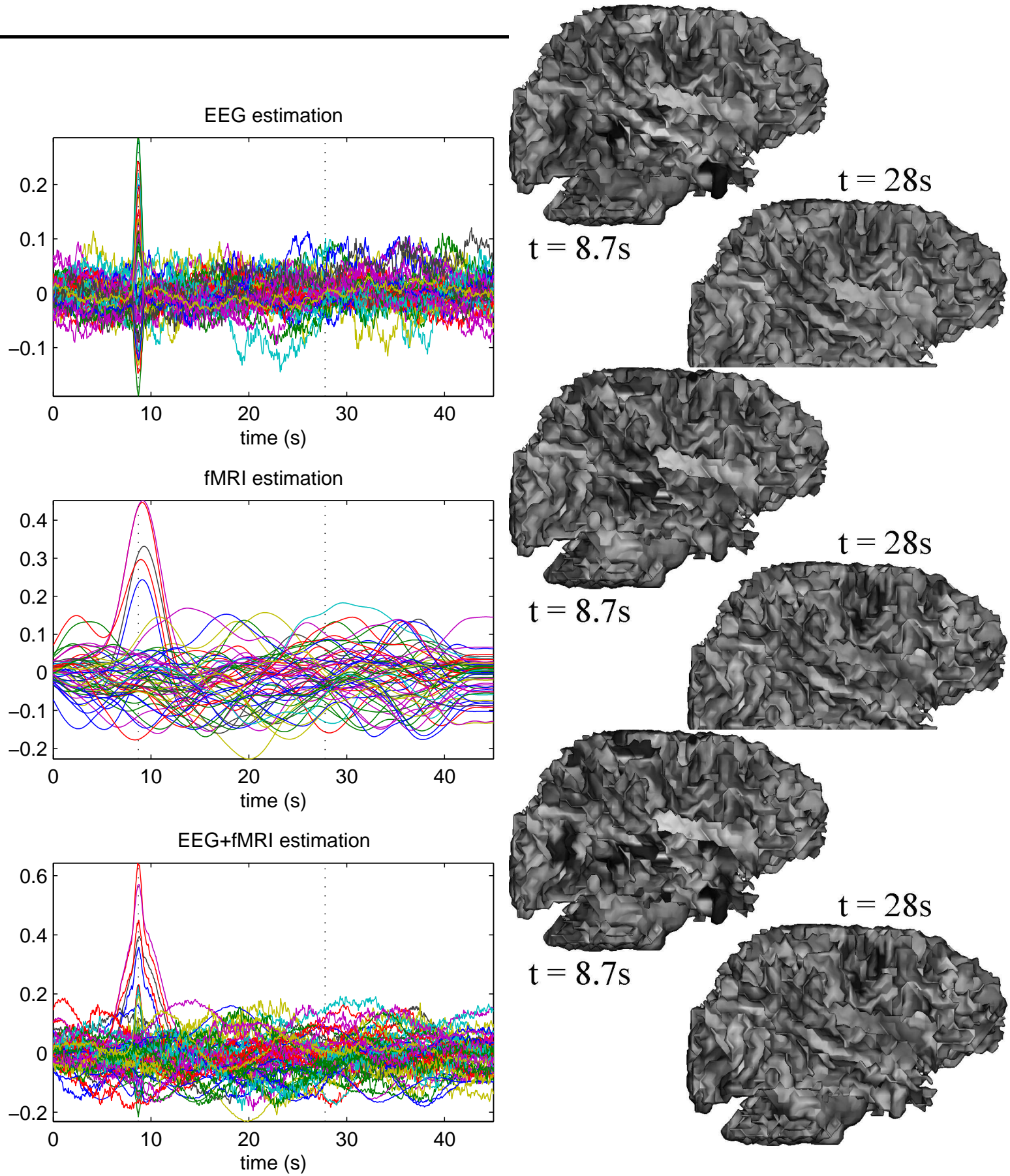

Figure 3: Estimated cortical activity when using the Kalman filter estimation on EEG and fMRI measures, both separately and together. Left: estimated cortical sources time courses. Right: estimated activities at two specific instants are mapped onto the cortex. 

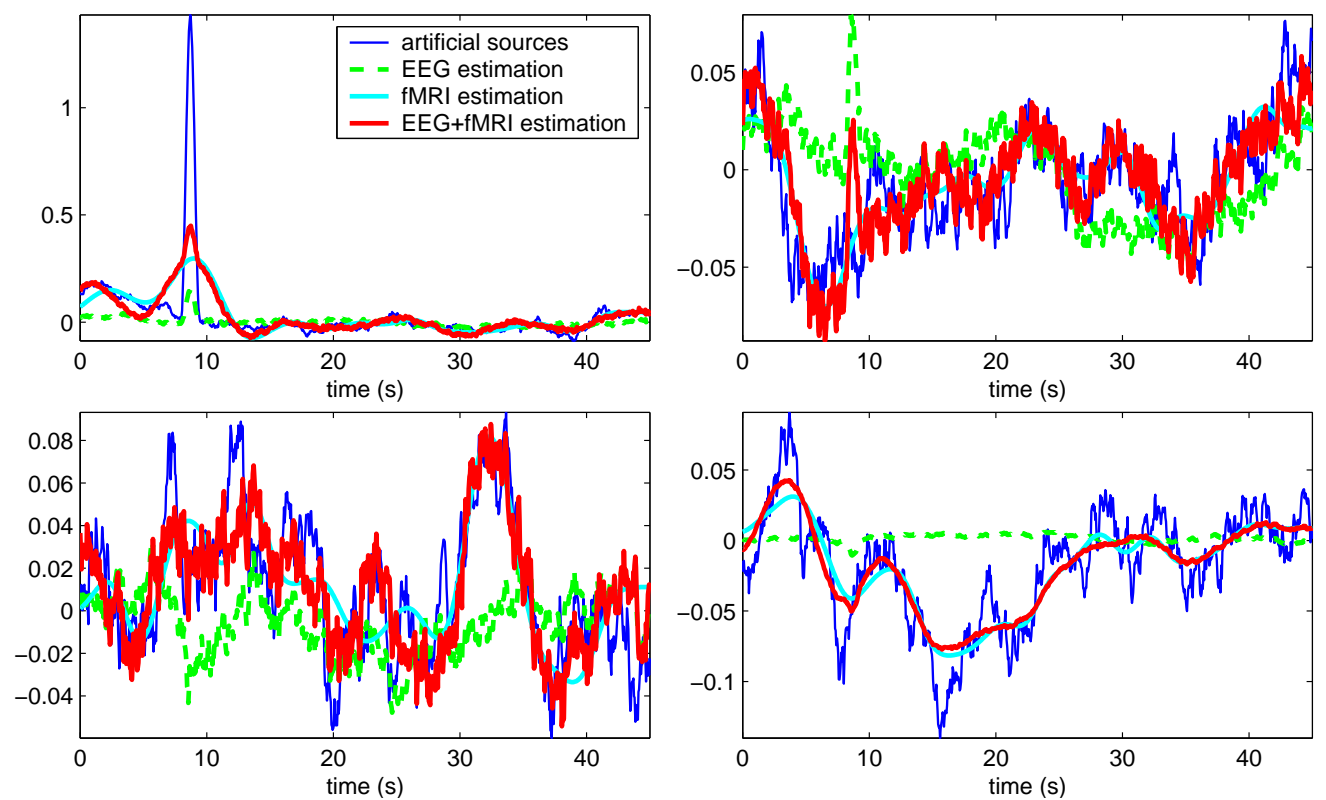

Figure 4: Estimated time courses of a few cortical sources are shown, and compared to their true values. Top: (left) fMRI alone was able to detect a spread out activity of an actually activated source; the fusion estimation is slightly more focal, but cannot violate more the smoothness constraint; (right) the inverse situation: EEG found activation in a non-activated source, and fMRI turns down this estimation. Bottom: (left) a typical example of how fMRI alone recovers the low frequency fluctuations, whereas EEG brings a complementary information on fast variations. (right) here, EEG was unable to give any information on the source activity; fusion estimation is then the same as that of fMRI alone.

and the actual link between the electrical activity implied in EEG and the metabolic energy demand implied in fMRI.

\section{Appendix}

The Kalman filter $(11 ; 12)$ is a recursive algorithm that alternatively computes the distribution of $X_{k}$ given measures before instant $k$ (evolution update step) and given measures until instant $k$ (measure update step), starting with the known a priori distribution of $X_{0}$ $\left(\hat{X}_{1}^{0}\right.$ and $\left.P_{1}^{0}\right)$. It requires a local linearization of the dynamical system at each instant.

Evolution update:

$$
\begin{aligned}
& \hat{X}_{k}^{k-1}=E\left(X_{k} \mid Y_{1} \ldots Y_{k-1}\right)=F\left(\hat{X}_{k-1}^{k-1}\right) \\
& P_{k}^{k-1}=E\left(X_{k} X_{k}^{T} \mid Y_{1} \ldots Y_{k-1}\right)=A P_{k-1}^{k-1} A^{T}+Q,
\end{aligned}
$$

where $A=\frac{\partial F}{\partial X}\left(\hat{X}_{k-1}^{k-1}\right)$. 
Measure update:

$$
\begin{aligned}
K & =P_{k}^{k-1} C^{T}\left(C P_{k}^{k-1} C^{T}+R\right)^{-1} \\
\hat{X}_{k}^{k} & =\hat{X}_{k}^{k-1}+K\left(Y_{k}-G\left(\hat{X}_{k}^{k-1}\right)\right) \\
P_{k}^{k} & =P_{k}^{k-1}-K C P_{k}^{k-1}
\end{aligned}
$$

where $C=\frac{\partial G}{\partial X}\left(\hat{X}_{k}^{k-1}\right)$.

After the Kalman filter forward pass, the Kalman smoother performs a backward pass, starting at $k=T-1$ :

$$
\begin{aligned}
J & =P_{k}^{k} A^{T}\left(P_{k+1}^{k}\right)^{-1} \\
\hat{X}_{k}^{T} & =\hat{X}_{k}^{k}+J\left(\hat{X}_{k+1}^{T}-\hat{X}_{k+1}^{k}\right) \\
P_{k}^{T} & =P_{k}^{k}+J\left(P_{k+1}^{T}-P_{k+1}^{k}\right) J^{T},
\end{aligned}
$$

where $A=\frac{\partial F}{\partial X}\left(\hat{X}_{k+1}^{T}\right)$.

\section{References}

[1] U. Hasson, Y. Nir, I. Levy, G. Fuhrmann, and R. Malach, "Intersubject synchronization of cortical activity during natural vision," Science, vol. 303, no. 5664, pp. 1634-1640, Mar 2004.

[2] C. Bénar, Y. Aghakhani, Y. Wang, A. Izenberg, A. Al-Asmi, F. Dubeau, and J. Gotman, "Quality of EEG in simultaneous EEG-fMRI for epilepsy," Clin Neurophysiol, vol. 114, no. 3, pp. 569-580, Mar 2003.

[3] B. Horwitz and D. Poeppel, "How can EEG/MEG and fMRI/PET data be combined?," Hum Brain Mapp, vol. 17, no. 1, pp. 1-3, Sep 2002.

[4] J. Mattout, M. Pelegrini-Issac, A. Bellio, J. Daunizeau, and H. Benali, "Localization Estimation Algorithm (LEA): a supervised prior-based approach for solving the EEG/MEG inverse problem," Inf Process Med Imaging, vol. 18, pp. 536-547, Jul 2003, Evaluation Studies.

[5] F. Babiloni, D. Mattia, C. Babiloni, L. Astolfi, S. Salinari, A. Basilisco, P. M. Rossini, M. G. Marciani, and Febo Cincotti, "Multimodal integration of EEG, MEG and fMRI data for the solution of the neuroimage puzzle," Magn Reson Imaging, vol. 22, no. 10, pp. 1471-1476, Dec 2004.

[6] P.-J. Lahaye, S. Baillet, J.-B. Poline, and L. Garnero, "Fusion of simultaneous fMRI/EEG data based on the electro-metabolic coupling," in Proc. 2th Proc. IEEE ISBI, Arlington, VA, Apr. 2004, pp. 864-867.

[7] G. Adde, M. Clerc, O. Faugeras, R. Keriven, J. Kybic, and T. Papadopoulo, "Symmetric BEM formulation for the M/EEG forward problem," in Proceedings of IPMI, Lecture Notes in Computer Science. 2003, pp. 524-535, Springer Verlag. 
[8] R. B. Buxton, K. UludaUg, D. J. Dubowitz, and T. T. Liu, "Modelling the hemodynamic response to brain activation," NeuroImage, vol. 23, pp. 220-233, 2004.

[9] J. Riera, J. Watanabe, I. Kazuki, M. Naoki, E. Aubert, T. Ozaki, and R. Kawashima, "A state-space model of the hemodynamic approach: nonlinear filtering of bold signals," NeuroImage, vol. 21, pp. 547-567, 2004.

[10] K. J. Friston, A. Mechelli, R. Turner, and C. J. Price, "Nonlinear responses in fmri : the balloon model, volterra kernels, and other hemodynamics," NeuroImage, vol. 12, pp. $466-477,2000$.

[11] M. Welling, Max Welling's Classnotes in Machine Learning, http://www.cs.toronto.edu/ $\sim$ welling/classnotes/papers_class/KF.ps.gz.

[12] C.K. Chui and G. Chen, Kalman Filtering with Real-Time Applications, Springer Ser. Info. Sci., Vol. 17. Springer, Berlin, Heidelberg, 1987.

\section{Contents}

1 Introduction $\quad 3$

2 Fusion Model $\quad 3$

3 Algorithm $\quad 5$

4 Artificial data $\quad 5$

5 Results $\quad 7$

$\begin{array}{llr}6 & \text { Conclusion } & 7\end{array}$

7 Appendix $\quad 9$

$\mathrm{RR} \mathrm{n}^{\circ} 5760$ 


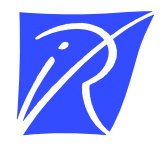

\section{Unité de recherche INRIA Sophia Antipolis 2004, route des Lucioles - BP 93 - 06902 Sophia Antipolis Cedex (France)}

Unité de recherche INRIA Futurs : Parc Club Orsay Université - ZAC des Vignes 4, rue Jacques Monod - 91893 ORSAY Cedex (France)

Unité de recherche INRIA Lorraine : LORIA, Technopôle de Nancy-Brabois - Campus scientifique 615, rue du Jardin Botanique - BP 101 - 54602 Villers-lès-Nancy Cedex (France)

Unité de recherche INRIA Rennes : IRISA, Campus universitaire de Beaulieu - 35042 Rennes Cedex (France)

Unité de recherche INRIA Rhône-Alpes : 655, avenue de l'Europe - 38334 Montbonnot Saint-Ismier (France)

Unité de recherche INRIA Rocquencourt : Domaine de Voluceau - Rocquencourt - BP 105 - 78153 Le Chesnay Cedex (France)

INRIA - Domaine de Voluceau - Rocquencourt, BP 105 - 78153 Le Chesnay Cedex (France)

http://www.inria.fr

ISSN 0249-6399 